\title{
Normal Criteria and Shared Values by Differential Polynomials"
}

\author{
Jihong Wang ${ }^{1}$, Qian Lü ${ }^{2}$, Qilong Liao ${ }^{3}$ \\ ${ }^{1}$ Department of Mathematics, Southwest University of Science and Technology, Mianyang, China \\ ${ }^{2}$ Department of Mathematics, Southwest University of Science and Technology, Mianyang, China \\ ${ }^{3}$ Department of Material Science and Engineer, Southwest University of Science and Technology, \\ Mianyang, China \\ E-mail:wangjihong@swust.edu.cn,luqiankuo1965@hotmail.com,liaoql@swust.edu.cn \\ Received April 5, 2011; revised April 28, 2011; accepted May 8, 2011
}

\begin{abstract}
For a family $F$ of meromorphic functions on a domain $D$, it is discussed whether $F$ is normal on $D$ if for every pair functions $f(z), g(z) \in F, f^{\prime}-a f^{n}$ and $g^{\prime}-a g^{n}$ share value $d$ on $D$ when $n=2,3$, where $a$, $b$ are two complex numbers, $a \neq 0, \infty, b \neq \infty$. Finally, the following result is obtained:Let $F$ be a family of meromorphic functions in $D$, all of whose poles have multiplicity at least 4 , all of whose zeros have multiplicity at least 2 . Suppose that there exist two functions $a(z)$ not idendtically equal to zero, $d(z)$ analytic in $D$, such that for each pair of functions $f$ and $g$ in $F, f^{\prime}-a(z) f^{2}$ and $g^{\prime}-a(z) g^{2}$ share the function $d(z)$. If $a(z)$ has only a multiple zeros and $f(z) \neq \infty$ whenever $a(z)=0$, then $F$ is normal in $D$.
\end{abstract}

Keywords: Normal Family, Meromorphic Function, Shared Value, Differential Polynomial

\section{Introduction and the Main Result}

In 1959,Hayman[4] proved

Theorem 1.1. Let $f$ be meromorphic functions in $C, n$ be a positive integer and $a, b$ be two constant such that $n \geq 5, a \neq 0, \infty$ and $b \neq \infty$. If

$$
f^{\prime}-a f^{n} \neq b
$$

then $f$ is a constant.

Corresponding to Theorem 1.1 there is the following theorems which confirmed a Hayman's well-known conjecture about normal families in [5].

Theorem 1.2. Let $F$ be a meromorphic function family in $D, n$ be a positive integer and $a, b$ be two constant such that $a \neq 0, \infty$ and $b \neq \infty$. If $n \geq 3$ and for each function $f \in F, \quad f^{\prime}-a f^{n} \neq b$, then $F$ is normal in $D$.

This result is due to S. Y. Li [8] $(n \geq 5), \mathrm{X}$. J. Li [9] $(n \geq 5)$, X. C. Pang $[10](n=4)$, H. H. Chen and M. L. Fang [2] $(n=3)$.

In 2001, M. L. Fang and W. J. Yuan [3] obtained

Theorem 1.3. Let $F$ be a meromorphic function family

"Supported by China Industrial Technology Development Program (B3120110001). in $D, a, b$ be two constants such that $a \neq 0, \infty$ and $b \neq \infty$. If, for each function $f \in F, f^{\prime}-a f^{2} \neq b$ and the poles of $f(z)$ are of multiplicity 3 at least, then $F$ is normal in $D$.

Let $D$ be a domain in $C, f(z)$ be meromorphic on $D$,and. $a \in C$

$$
E_{f}(a)=f^{-1}(a) \cap D=\{Z \in D: f(z)=a\}
$$

Two functions $f$ and $g$ are said to share the value $a$ if $E_{f}(a)=E g(a)$. For a case $n \geq 4$ in Theorem 1.2, Q. C. Zhang [14] improved Theorem 1.2 by the idea of shared values and obtained the following result.

Theorem 1.4. Let $F$ be a family of meromorphic functions in $D, n$ be a positive integer and $a, b$ be two constant such that $n \geq 4, a \neq 0, \infty$ and $b \neq \infty$. If, for each pair of functions $f$ and $g$ in $F, f^{\prime}-a f^{n}$ and $g^{\prime}-a g^{n}$ share the value $b$, then $F$ is normal in $D$.

In this paper, we shall discuss a condition on which $F$ still is normal in $D$ for the case $2 \leq n \leq 3$ and obtain the following result.

Theorem 1.5. Let $F$ be a family of meromorphic functions in $D$, all of whose poles have multiplicity 2 at least, and $a, b$ be two constant such that $a \neq 0, \infty$ and $b \neq \infty$. 
If, for each pair of functions $f$ and $g$ in $F, f^{\prime}-a f^{3}$ and $g^{\prime}-a g^{3}$ share the value $b$ in $D$, then $F$ is normal in $D$.

We denote $f^{\#}(z)=\frac{\left|f^{\prime}(z)\right|}{1+|f(z)|^{2}}$ for the spherical derivatives of $f(z)$. The following example imply that the restriction of poles in Theorem 1.5 is necessary.

Example 1. [14] Let $D=\{z:|z|<1\}$ and $F=\left\{f_{n}\right\}$, where

$$
f_{n}(z)=\frac{1}{\sqrt{n}(z-1 / n)}, z \in D, n=1,2, \cdots
$$

Then for each pair $m, n, f_{m}^{\prime}-f_{m}^{3}$ and $f_{n}^{\prime}-f_{n}^{3}$ share the value 0 in $D$. But $F$ is not normal at $z=0$ since $f_{n}^{\#}(1 / \sqrt{n}) \rightarrow \infty$.

But we also have the following examples which imply that on the same as restriction of poles in Theorem $1.5 \mathrm{~F}$ is not normal in $D$ if for each pair of functions $f$ and $g$ in $F, f^{\prime}-a f^{2}$ and $g^{\prime}-a g^{2}$ share the value $b$ on $D$.

Example 2. [3] Let $f_{n}(z)=n z /(z \sqrt{n}-1)^{2}$ for $n=1,2, \cdots$, and $\Delta=\{z:|z|<1\}$ Clearly,

$$
f_{n}^{\prime}(z)+f_{n}^{2}=n(z \sqrt{n}-1)^{-4} \neq 0,
$$

and $f_{n}(z)$ only a double pole and a simple zero. Since $f_{n}^{\#}(0)=n \rightarrow \infty$, as $n \rightarrow \infty$ from Marty's criterion we have that $\left\{f_{n}(z)\right\}$ is not normal in $\Delta$ In fact, in thepresent paper we also obtain two results as follows.

Theorem 1.6. Let $F$ be a family of meromorphic functions in D, all of whose poles have multiplicity 4 at least, all of whose zeros have multiplicity 2 at least, and $a, b$ be two constant such that $a \neq 0, \infty$ and $b \neq \infty$. If, for each pair of functions $f$ and $g$ in $F, f^{\prime}-a f^{2}$ and $g^{\prime}-a g^{2}$ share the value $b$ in $D$, then $F$ is normal in $D$.

Theorem 1.7. Let $F$ be a family of meromorphic functions in D, all of whose poles have multiplicity at least 4 , all of whose zeros have multiplicity at least 2. Suppose that there exist two functions $a(z)$ not idendtically equal to zero, $d(z)$ analytic in $D$, such that for each pair of functions $f$ and $g$ in $F, f^{\prime}-a(z) f^{2}$ and $g^{\prime}-a(z) g^{2}$ share the function $d(z)$ in D. If $a(z)$ has only a multiple zeros and $f(z) \neq \infty$ whenever $a(z)=0$ then $F$ is normal in $D$.

The following example shows that the condition $f(z) \neq \infty$ when $a(z)=0$ in Theorem 1.7 is necessary.

Example 3. [7] Let $D=\{z:|z|<1\}$ and $F=\left\{f_{n}\right\}$ where $f_{n}(z)=\frac{1}{n z^{4}}, z \in D, n=1,2, \cdots$. We take $a(z)=-4 z^{3}$ and $d(z) \equiv 0$. Clearly, $F$ fails to be normal at $z=0$ However, all poles of $f_{n}(z)$ are of multiplicity 4 , and for each pair $m, n, f_{m}^{\prime}-a(z) f_{m}{ }^{2}$ and $f_{n}^{\prime}-a(z) f_{n}^{2}$ share analytic functions $d(z)$ in $\Delta$.

\section{Lemmas}

To prove the above theorems, we need some lemma as follows:

Lemma 2.1. ([1,2]) Let $f(z)$ be a meromorphic function in $C, n$ be a positive integer and $b$ be a non-zero constant. If $f^{n} f^{\prime} \neq b$, then $f$ is a constant. Moreover if $f$ is a transcendental meromorphic function, then $f^{n} f^{\prime}(z)$ assumes every finite non-zero value finitely often.

Lemma 2.2. ([1]) Let $f(z)$ be a transcendental meromorphic function with finite order in $C$. If $f(z)$ has only multiple zeros, then it's first derivative $f^{\prime}$ assumes every finite value except possibly zero infinitely often.

Lemma 2.3. ([12]) Let $f(z)$ be a non-polynomials rational function in C. If $f(z)$ has only zeros of multiplicity 2 at least, then $f=\frac{(c z+d)^{2}}{a z+b}$ where $a, b, c, d$ are four constants, $a \neq 0, c \neq 0$.

Lemma 2.4. ([4]) If $f(z)$ be a transcendental meromorphic function in $C$, then either $f(z)$ assumes every finite value infinitely often or every derivative $f^{(l)}$ assumes every finite value except possibly zero infinitely often. If $f(z)$ is a non-constant rational function and $f(z) \neq a$, a is a finite value, then $f^{(l)}$ assumes every finite value except possibly zero at least once.

Lemma 2.5. ([11]) Let $f(z)$ be a transcendental meromorphic function with finite order, all of whose zeroes are of multiplicity at least $k+1$, and let $P(z)$ be a polynomial, $P(z)$ is not idendtically equal to zero. Then $f^{(k)}(z)-P(z)$ has infinitely many zeros often.

Lemma 2.6. ([6]) Let $f(z)$ be a non-polynomial rational functions in $\mathrm{C}$, all of whose zeroes are of multiplicity at least 4 . Then $f^{\prime}(z)-z^{r}$ has a zeros at least often.

Lemma 2.7. ([13]) Let $F$ be a family of meromorphic functions on the unit disc $\Delta$, all of whose zeroes have multiplicity $p$ at least, all of whose poles have multiplicity $q$ at least. Let $\alpha$ be a real number satisfying $-p<\alpha<q$. Then $F$ is not normal at a point $z_{0} \in \Delta$ if and only if there exist

1) points $z_{n} \in \Delta, z_{n} \rightarrow z_{0}$

2) functions $f_{n} \in F$; and

3) positive numbers $\rho_{n} \rightarrow 0$

such that 


$$
\rho_{n}^{\alpha} f_{n}\left(z_{n}+\rho_{n} \xi\right)=g_{n}(\xi) \rightarrow g(\xi)
$$

spherically uniformly on each compact subset of $C$, where $g(\xi)$ is a non-constant meromorphic function satisfying the zeros of $g(\xi)$ are of multiplicities $p$ at least and the poles of $g(\xi)$ are of multiplicities $q$ at least. Moreover, the order of $g(\xi)$ is not greater than 2 .

\section{Proofs of Theorem 1.5.-1.7.}

\subsection{Proof of Theorem 1.5.}

Suppose that there exists one point $z_{0} \in D$ such that $F$ is not normal at point $z_{0}$. Without loss of generality we assume that $z_{0}=0$. By Lemma 2.7, there exist points, $z_{n} \in \Delta, z_{n} \rightarrow z_{0}$, functions $f_{n} \in F$ and positive numbers $\rho_{n} \rightarrow 0$ such that

$$
g_{j}(\xi)=\rho_{j}^{\frac{1}{n-1}} f_{j}\left(z_{j}+\rho_{j} \xi\right) \Rightarrow g(\xi)
$$

spherically uniformly on each compact subset of $C$, where $g(\xi)$ is a non-constant meromorphic function with order $\leq 2$, all of whose poles are of multiplicities $\mathrm{k}$ at least.

From (3.1) we have

$$
\begin{aligned}
& \rho_{j}^{\frac{n}{n-1}}\left\{f_{j}^{\prime}\left(z_{j}+\rho_{j} \xi\right)-a f_{j}^{n}\left(z_{j}+\rho_{j} \xi\right)-b\right\} \\
& =g_{j}^{\prime}(\xi)-a g_{j}^{n}(\xi)-\rho_{j}^{\frac{n}{n-1}} b \Rightarrow g^{\prime}(\xi)-a g^{n}
\end{aligned}
$$

By the same method as [14], from Lemma 2.1 it is not difficult to find that $g^{\prime}-a g^{n}$ has just a unique zero $\xi=\xi_{0}$.

Set $g=1 / \varphi$ again, if $n \geq 3$ then

$$
g^{\prime}-a g^{n}=-\left[\varphi^{\prime} \varphi^{n-2}+a\right] / \varphi^{n}
$$

thus $\left[\varphi^{\prime} \varphi^{n-2}+a\right] / \varphi^{n}$ has just a unique zero $\xi=\xi_{0}$. Thus $\xi_{0}$ is a multiple pole of $\varphi$ or else a zero of $\varphi^{\prime} \varphi^{n-2}+a$.

If $\xi_{0}$ is a multiple pole of $\varphi$, since

$$
\left[\varphi^{\prime} \varphi^{n-2}+a\right] / \varphi^{n}
$$

has only one zero $\xi_{0}$, then $\varphi^{\prime} \varphi^{n-2}+a \neq 0$. By Lemma 2.1 again, $\varphi$ is a constant which contradicts with $g$ is not any constant.

So we have that $\varphi$ has no multiple poles and $\varphi^{\prime} \varphi^{\prime}+a$ have only a unique zero. By Lemma 2.1 , and Lemma 2.4, we have $\varphi$ is not transcendental.

If $\varphi$ is non-constant polynomial, then

$$
\varphi^{\prime} \varphi^{n-2}+a=A\left(\xi-\xi_{0}\right)^{l} .
$$

Since all zeros of $\psi$ are of multiplicity 2 , then $l \geq 3$. Denoting $\psi$ for $\varphi^{n-1} /(n-1), \psi=\varphi^{n-1} /(n-1)$, we have $\psi^{\prime}=A\left(\xi-\xi_{0}\right)^{l}-a$ and $\psi^{\prime \prime}=A l\left(\xi-\xi_{0}\right)^{l-1}$. Since all zeros of $\varphi$ are of multiplicity $2(n-1) \geq 4$, then $\psi(\xi) \neq 0, \xi \neq \xi_{0}$.

If $\psi\left(\xi_{0}\right)=0$, then $\psi^{\prime}\left(\xi_{0}\right)=0$ which contradicts with $\psi^{\prime}\left(\xi_{0}\right)=-a \neq 0$. So $\psi$ is a constant.

Next we prove that there exists no rational functions such as $\psi$. Noting that $\psi=\varphi^{n-1} /(n-1)$ and $\psi$ has no multiple pole, we may set

$$
\psi(\xi)=A \frac{\left(\xi-\xi_{1}\right)^{m_{1}}\left(\xi-\xi_{2}\right)^{m_{2}} \cdots\left(\xi-\xi_{s}\right)^{m_{s}}}{\left(\eta-\eta_{1}\right)^{n-1}\left(\eta-\eta_{2}\right)^{n-1} \cdots\left(\eta-\eta_{t}\right)^{n-1}},
$$

where $A$ is a non-zero constant, $s \geq 1, t \geq 1, m_{1}, m_{2}, \cdots, m_{s}$ are $s$ positive integers, $m_{j} \geq 2(n-1),(j=1,2, \cdots, s)$. For a convenience of stating, we denote

$$
m=m_{1}+m_{2}+\cdots+m_{s},
$$

then $m \geq 2(n-1) s$.

From (3.3), we have

$$
\psi^{\prime}(\xi)=A \frac{\left(\xi-\xi_{1}\right)^{m_{1}-1} \cdots\left(\xi-\xi_{s}\right)^{m_{s}-1} h(\xi)}{\left(\eta-\eta_{1}\right)^{n} \cdots\left(\eta-\eta_{t}\right)^{n}}=\frac{p_{1}(\xi)}{q_{1}(\xi)},
$$

where

$$
\begin{gathered}
h(\xi)=[m-t(n-1)] \xi^{s+t-1}+a_{s+t-2} \xi^{s+t-2}+\cdots+a_{0} \\
p_{1}(\xi)=\left(\xi-\xi_{1}\right)^{m_{1}-1} \cdots\left(\xi-\xi_{s}\right)^{m_{s}-1} h(\xi) \\
q_{1}(\xi)=\left(\eta-\eta_{1}\right)^{n} \cdots\left(\eta-\eta_{t}\right)^{n}
\end{gathered}
$$

are three polynomials. Since $\psi^{\prime}(\xi)+a$ has only a unique zero $\xi_{0}$ then there exists a non-zero constant $B$ such that

$$
\psi^{\prime}(\xi)+a=\frac{B\left(\xi-\xi_{0}\right)^{l}}{\left(\eta-\eta_{1}\right)^{n}\left(\eta-\eta_{2}\right)^{n} \cdots\left(\eta-\eta_{t}\right)^{n}},
$$

so

$$
\psi^{\prime \prime}(\xi)=\frac{B\left(\xi-\xi_{0}\right)^{l-1} p_{2}(\xi)}{\left(\eta-\eta_{1}\right)^{n+1}\left(\eta-\eta_{2}\right)^{n+1} \cdots\left(\eta-\eta_{t}\right)^{n+1}},
$$

where $p_{2}(\xi)=(l-n t) \xi^{t}+b_{t-1} \xi^{t-1}+\cdots+b_{0}$ is a polynomial. From (3.5) we also have

$$
\psi^{\prime \prime}(\xi)=A \frac{\left(\xi-\xi_{1}\right)^{m_{1}-2} \cdots\left(\xi-\xi_{s}\right)^{m_{s}-2} p_{3}(\xi)}{\left(\eta-\eta_{1}\right)^{n+1} \cdots\left(\eta-\eta_{t}\right)^{n+1}}
$$

where $p_{3}(\xi)$ is a polynomial also.

We denote $\operatorname{deg}(p)$ for the degree of a polynomial $p(\xi)$, from (3.5) and (3.6) we may obtain

$$
\begin{aligned}
& \operatorname{deg}(h) \leq s+t+1 \\
& \operatorname{deg}\left(p_{1}\right) \leq m+t+1, \quad \operatorname{deg}\left(q_{1}\right)=n t
\end{aligned}
$$


From (3.8), (3.9) and (3.10) we may obtain

$$
\begin{gathered}
\operatorname{deg}\left(p_{2}\right) \leq t, \\
\operatorname{deg}\left(p_{3}\right) \leq 2 t+2 s-2 .
\end{gathered}
$$

Since $\psi^{\prime}(\xi)+a$ has only a unique zero $\xi=\xi_{0}$ and

$$
m_{j}-2 \geq 1 \quad(j=1,2, \cdots, s),
$$

then $\xi_{0} \neq \xi_{j} \quad(j=1,2, \cdots, s)$. From (3.8), (3.9) and (3.11) it follows that $\operatorname{deg}\left(p_{3}(\xi)\right) \geq l-1$ then

$$
m-2 s \leq \operatorname{deg}\left(p_{2}\right) \leq t,
$$

Since $m_{j} \geq 2(n-1)$, then $m \geq 2(n-1) s$, so by (3.13) we have $2 s \leq t$.

If $l \geq n t$, from (3.8), (3.9) and (3.12), we have

$$
n t-1 \leq l-1 \leq \operatorname{deg}\left(p_{3}\right) \leq 2 t+2 s-2
$$

Then, $t \leq 2 s-1$. Combining with above inequality $2 s \leq t$, we bring about a contradiction.

If $l<n t$, then from (3.5) and (3.7) we have

$$
\operatorname{deg}\left(p_{1}\right)=\operatorname{deg}\left(q_{1}\right)
$$

that is $m-s+\operatorname{deg}(h)=n t$. If $m=t(n-1)$, then $\operatorname{deg}(h) \leq s+t-2$. So

$$
\begin{aligned}
m-t(n-1) & =s+n t-\operatorname{deg}(h)-t(n-1) \\
& =s+t-\operatorname{deg}(h) \\
& \geq s+t(s+t-2)=2
\end{aligned}
$$

this is impossible. Thus, $m \neq t(n-1)$ and $\operatorname{deg}(h)=s+t-1$. Therefore, $m=1+t(n-1)$. Again from (3.8) and (3.9), we have $m-2 s \leq t$. Then $t \leq 2 s-1$, this contradicts to $2 s \leq t$.

This completes the proof of Theorem 1.5.

\subsection{Proof of Theorem 1.6.}

For any points $z_{0} \in D$, Without loss of generality, we set $z_{0}=0$. Suppose that $F$ is not normal at $z_{0}=0$, then by Lemma 2.7 , we have that there exist a subsequence $f_{n} \subset F$, points sequence $z_{0} \in D$, and a positive numbers $\rho_{n}, \rho_{n} \rightarrow 0^{+}$, such that

$$
g_{n}(\xi)=1 / \rho_{n} \quad f_{n}\left(z_{n}+\rho_{n} \xi\right) \rightarrow g(\xi),
$$

spherically uniformly on each compact subset of $C$, where $g(\xi)$ is a non-constant meromorphic function with order $\leq 2$, all of whose poles are ofmultiplicities at least 2, all of whose zeros are of multiplicities at least 4.

From (3.14) we have

$$
\frac{1}{g_{n}^{2}(\xi)}\left(g_{n}^{\prime}(\xi)+a\right)+\rho_{n}^{2} d \rightarrow \frac{g^{\prime}+a}{g^{2}(\xi)}
$$

If $g^{\prime}(\xi)+a \equiv 0$, then $g(\xi)=-a \xi+c_{0}$, this contra- dicts to which all zeros of $g(\xi)$ have multiplicity at least 4. If for any point $\xi \in C, g^{\prime}(\xi)+a \neq 0$, then By Lemma 2.2, we have that $g(\xi)$ is not transcendental in $C$, so $g(\xi)$ is non-constant rational function in $C$. By Lemma 2.3 we also have that

$$
g(\xi)=\frac{(c \xi+d)^{3}}{a \xi+b}
$$

a contradictions. Therefore, $\left[g^{\prime}(\xi)+a\right] / g^{2}(\xi)$ have a zeros. We may claim that $\left[g^{\prime}(\xi)+a\right] / g^{2}(\xi)$ has a unique zero $\xi+\xi_{0}$. Otherwise, suppose that $\xi_{0}, \xi_{0}^{*}$ are two distinguish zeros of

$$
\left[g^{\prime}(\xi)+a\right] / g^{2}(\xi)
$$

then there exists a positive number $\delta>0$ such that $N\left(\xi_{0}, \delta\right) \cap N\left(\xi_{0}^{*}, \delta\right)=\phi$.On the other hand, by Hurwitz's Theorem we can find two point sequences $\xi_{n} \in N\left(\xi_{0}, \delta\right), \quad \xi_{n}^{*} \in N\left(\xi_{0}^{*}, \delta\right)$ Such that $\xi_{n} \rightarrow \xi_{0}, \xi_{n}^{*} \rightarrow \xi_{0}^{*}$, and

$$
\begin{aligned}
& g_{n}^{-2}\left(\xi_{n}\right)\left[g_{n}^{\prime}\left(\xi_{n}\right)+a\right]+\rho_{n}^{2} d=0 \\
& g_{m}^{-2}\left(\xi_{m}^{*}\right)\left[g_{m}^{\prime}\left(\xi_{m}^{*}\right)+a\right]+\rho_{m}^{2} d=0
\end{aligned}
$$

then, we have

$$
\begin{gathered}
f_{n}^{\prime}\left(z_{n}+\rho_{n} \xi_{n}\right)-a f_{n}^{2}\left(z_{n}+\rho_{n} \xi_{n}\right)-d=0, \\
f_{m}^{\prime}\left(z_{m}+\rho_{m} \xi_{m}^{*}\right)-a f_{m}^{2}\left(z_{m}+\rho_{m} \xi_{m}^{*}\right)-d=0 .
\end{gathered}
$$

From the hypothesis that for every pair functions $f$, $g$ in $F, f^{\prime}(z)-a f^{2}$ and $g^{\prime}(z)-a g^{2}$ share complex number $d$ in $D$, we have

$$
\begin{aligned}
& f_{m}^{\prime}\left(z_{n}+\rho_{n} \xi_{n}\right)-a f_{n}^{2}\left(z_{n}+\rho_{n} \xi_{n}\right)-d=0, \\
& f_{m}^{\prime}\left(z_{n}+\rho_{n} \xi_{n}^{*}\right)-a f_{m}^{2}\left(z_{n}+\rho_{n} \xi_{n}^{*}\right)-d=0 .
\end{aligned}
$$

Fix $m$, let $n \rightarrow \infty$, then $f_{m}^{\prime}(0)-a f_{m}^{2}(0)-d=0$.

Since $f_{m}^{\prime}(z)-a f_{m}^{2}(z)-d$ has no accumulation points, so for sufficiently large $n$ we have

$$
z_{n}+\rho_{n} \xi_{n}=0, z_{m}+\rho_{m} \xi_{m}^{*}=0
$$

then

$$
\xi_{n}=-\frac{z_{n}}{\rho_{n}}, \xi_{n}^{*}=-\frac{z_{n}}{\rho_{n}}
$$

This contradicts to $N\left(\xi_{0}, \delta\right) \cap N\left(\xi_{0}^{*}, \delta\right)=\phi$. Thus, $\left[g^{\prime}(\xi)+a\right] / g^{2}(\xi)$ has a unique zero $\xi=\xi_{0}$. Furthermore, we have that either $\xi=\xi_{0}$ is a multiple poles of $g(\xi)$ or $\xi=\xi_{0}$ is a unique zero of $g^{\prime}(\xi)+a$. If $\xi=\xi_{0}$ is a multiple poles of $g(\xi)$, then $g^{\prime}(\xi)+a \neq 0$, for any $\xi \in C$. By Lemma 2.2 and Lemma 2.3, we immediately deduce that $g(\xi)$ must be a constant in $C$, 
which contradicts to $g(\xi)$ is a non-constant meromorphic functions in $C$. Therefore, $g(\xi)$ has only a simple poles and $g^{\prime}(\xi)+a$ has a unique $\xi=\xi_{0}$. But since $g(\xi)$ has only a multiple poles, so we have that $g(\xi)$ is entire in $C$ and $g^{\prime}(\xi)+a$ has a unique $\xi=\xi_{0}$. Also by Lemma 2.2, we have that $g(\xi)$ is a non-constant polynomials, all of whose zeros are of multiplicity at least 4 . Setting

$$
g(\xi)=A\left(\xi-\xi_{1}\right)^{m_{1}}\left(\xi-\xi_{2}\right)^{m_{2}} \cdots\left(\xi-\xi_{s}\right)^{m_{s}},
$$

we have

$$
g^{\prime}(\xi)=A\left(\xi-\xi_{1}\right)^{m_{1}-1}\left(\xi-\xi_{2}\right)^{m_{2}-1} \cdots\left(\xi-\xi_{2}\right)^{m_{s}-1} h(\xi)
$$

Where $h(\xi)=m \xi^{s-1}+a_{0} \xi^{s-2}+\cdots+a_{s-2}, A \neq 0$, $a_{0}, a_{1}, \cdots, a_{s-2}$ are some complex constants,

$m_{j}(j=1,2, \cdots, s)$ are $s$ positive integers, $m_{j} \geq 4$, and $m=\sum_{j=1}^{j=s} m_{j}$. Thus, we have

$$
g^{\prime}(\xi)+a=B\left(\xi-\xi_{0}\right)^{l},
$$

where $l \geq 3$. So we have that $g^{\prime \prime}(\xi)+a=B l\left(\xi-\xi_{0}\right)^{l-1}$.

If $g\left(\xi_{0}\right)=0$, then $g^{\prime}\left(\xi_{0}\right)=g^{\prime \prime}\left(\xi_{0}\right)=g^{\prime \prime \prime}\left(\xi_{0}\right)=0$.

But $g^{\prime}\left(\xi_{0}\right)=-a \neq 0$, a contradictions.

Therefore, $F$ is normal at $z=0$.

\subsection{Proof of Theorem 1.7.}

For any $z \in D$, if $a(z) \neq 0$, we may give the complete proof of Theorem 1.7 by the same argument as Theorem 1.6 , we emit the detail. In the sequel, we shall prove that $F$ is normal at which $a(z)=0$. Set $a(z)=z^{r} b(z)$, where $b(z)$ is analytic at $z=0, b(0)=1, r$ is a positive integer, $r \geq 2$.

$$
F_{1}=\left\{F: F(z)=\frac{1}{z^{r} f(z)}, f(z) \in F\right\}
$$

For every function $F(z)$ in $F_{1}$, from the hypothesis in Theorem 1.7, we can see that all zeros of $F(z)$ are of order at least 4, all poles of $F(z)$ are of multiplicity at least 2.

Suppose that $F_{1}$ is not normal at $z=0$, then by Lemma 2.7, there exists a subsequence $F_{n} \subset F_{1}$, a point sequence $z_{n},\left|z_{n}\right|<r<1$, and a positive number sequence $\rho_{n}, \rho_{n} \rightarrow 0^{+}$, such that

$$
\begin{aligned}
g_{n}(\xi) & =\rho_{n}^{-1} F_{n}\left(z_{n}+\rho_{n} \xi\right) \\
& =\rho_{n}^{-1}\left(z_{n}+\rho_{n} \xi\right)^{-r} f_{n}^{-1}\left(z_{n}+\rho_{n} \xi\right) \\
& \rightarrow g(\xi)
\end{aligned}
$$

spherically uniformly on compact subsets of $C$, where $g(\xi)$ is a non-constant meromorphic function on $C$, all of whose zeros are of multiplicity at least 4 , and all of whose poles are multiple. Moreover, $g(\xi)$ has an order at most 2.

Now we distinguish two cases:

Case 1. $z_{n} / \rho_{n} \rightarrow \infty$. Without loss of a generalization, we assume that there exists a point $z^{\prime}$ such that $z_{n} \rightarrow z^{\prime},\left|z^{\prime}\right| \leq r \leq 1$, we have

$$
\begin{aligned}
& f_{n}^{\prime}\left(z_{n}+\rho_{n} \xi\right) \\
& =-\frac{g_{n}^{\prime}(\xi)}{\rho_{n}^{2}\left(z_{n}+\rho_{n} \xi\right)^{r} g_{n}^{2}(\xi)}-\frac{r}{\rho_{n}\left(z_{n}+\rho_{n} \xi\right)^{r+1} g_{n}(\xi)} \\
& =-\frac{1}{\rho_{n}^{2}\left(z_{n}+\rho_{n}\right)^{r}}\left\{\frac{g_{n}^{\prime}}{g_{n}^{2}}+r\left(\frac{z_{n}}{\rho_{n}}+\xi\right)^{-1} \cdot \frac{1}{g_{n}(\xi)}\right\}
\end{aligned}
$$

For the sake of convenience, we denote $S_{1}$ for the set of all zeros of $g(\xi), S_{2}$ for the set of all zeros of $g^{\prime}(\xi)$, and $S_{3}$ for the set of all poles of $g(\xi)$.

Since $\lim _{n \rightarrow+\infty} \frac{g_{n}^{\prime}(\xi)}{g_{n}^{2}(\xi)}=\frac{g^{\prime}(\xi)}{g^{2}(\xi)}, \lim _{n \rightarrow+\infty} \frac{1}{g_{n}(\xi)}=\frac{1}{g(\xi)}$ uniformly on compact subsets of $C \backslash S_{1}$, and $\lim _{n \rightarrow+\infty} \frac{r}{z_{n} /\left(\rho_{n}+\xi\right)}=0$ uniformly on compact subsets of $C$, thus $\lim _{n \rightarrow \infty} f_{n}\left(z_{n}+\rho_{n} \xi\right)=\infty$, uniformly on compact subsets of $C \backslash\left(S_{1} \cup S_{2} \cup S_{3}\right)$. Thus, it is not difficult to see that

$$
\begin{aligned}
& \frac{f_{n}^{\prime}\left(z_{n}+\rho_{n} \xi\right)-a\left(z_{n}+\rho_{n} \xi\right) f_{n}^{2}\left(z_{n}+\rho_{n} \xi\right)}{a\left(z_{n}+\rho_{n} \xi\right) f_{n}^{2}\left(z_{n}+\rho_{n} \xi\right)-d\left(z_{n}+\rho_{n} \xi\right)} \\
& -\frac{d\left(z_{n}+\rho_{n} \xi\right)}{a\left(z_{n}+\rho_{n} \xi\right) f_{n}^{2}\left(z_{n}+\rho_{n} \xi\right)-d\left(z_{n}+\rho_{n} \xi\right)} \\
& \rightarrow-\frac{g^{\prime}(\xi)}{b\left(z^{\prime}\right)}-1
\end{aligned}
$$

uniformly on compact subsets of $C \backslash\left(S_{1} \cup S_{2} \cup S_{3}\right)$. If $-\frac{g^{\prime}(\xi)}{b\left(z^{\prime}\right)}-1 \neq 0$, then $g^{\prime}(\xi) \neq-b\left(z^{\prime}\right)$, for any

$\xi \in C \backslash\left(S_{1} \cup S_{2} \cup S_{3}\right)$. Thus, $g^{\prime}(\xi) \neq-b\left(z^{\prime}\right)$ for any $\xi \in C$. By Lemma 2.5, we can see that $g(\xi)$ is not transcendental in $\mathrm{C}$, but is a rational function. Also from Lemma 2.3, we deduce that $g(\xi)$ is constant, which contradicts to the fact that $g(\xi)$ is non-constant. On the other hand, it is easy to see that $g^{\prime}(\xi)$ is not identically equal to $-b\left(z^{\prime}\right)$. Hence, $g^{\prime}(\xi)+b\left(z^{\prime}\right)$ has one zeros at least in $C$. In fact, by the same as the arguments in Theorem 1.5 and Theorem 1.6, we deduce that $g(\xi)$ has a unique zero $\xi=\xi_{0}$. By Lemma 2.5 , we can see that $g(\xi)$ is not transcendental in $C$, so $g(\xi)$ is nonconstant rational function in $C$. For a non-constant poly- 
nomials $g(\xi)$, and noting that $g(\xi)$ has only a zero with multiplicity at least 4 , we have

$$
g^{\prime}(\xi)+b\left(z^{\prime}\right)=B\left(\xi-\xi_{0}\right)^{l}, \quad l \geq 3
$$

Thus, $g^{\prime \prime}(\xi)=B l\left(\xi-\xi_{0}\right)^{l-1}$. Hence, $g(\xi)$ has a zero $\xi=\xi_{0}$ at most. If $\xi=\xi_{0}$ is a zero of $g(\xi)$, then $g^{\prime}\left(\xi_{0}\right)=g^{\prime \prime}\left(\xi_{0}\right)=g^{\prime \prime \prime}\left(\xi_{0}\right)=0$. But $g^{\prime}\left(\xi_{0}\right)=-b\left(z^{\prime}\right) \neq 0$, a contradiction.

In the sequel, we denote $\operatorname{deg}(p)$ for the degree of a polynomial $p(\xi)$. If $g(\xi)$ is non polynomials rational functions, then we set

$$
g(\xi)=A \frac{\left(\xi-\xi_{1}\right)^{m_{11}}\left(\xi-\xi_{2}\right)^{m_{2}} \cdots\left(\xi-\xi_{s}\right)^{m_{s}}}{\left(\xi-\eta_{1}\right)^{n_{1}}\left(\xi-\eta_{2}\right)^{n_{2}} \cdots\left(\xi-\eta_{t}\right)^{n_{t}}},
$$

Where $m_{j} \geq 4, j=1,2, \cdots, s ; n_{j} \geq 2, j=1,2, \cdots, t$.

$$
m=\sum_{j=1}^{s} m_{j} \geq 4 s, q=\sum_{k=1}^{t} n_{k} \geq 2 t
$$

Then,

$$
g^{\prime}(\xi)=\frac{p_{1}(\xi)}{q_{1}(\xi)}=\frac{A\left(\xi-\xi_{1}\right)^{m_{1}-1} \cdots\left(\xi-\xi_{s}\right)^{m_{s}-1} h(\xi)}{\left(\xi-\eta_{1}\right)^{n_{1}+1} \cdots\left(\xi-\eta_{t}\right)^{n_{t}+1}}
$$

where

$$
\begin{gathered}
h(\xi)=(m-q) \xi^{s+t-1}+a_{0} \xi^{s+t-2}+\cdots+a_{s+t-2}, \\
\operatorname{deg}(h) \leq s+t-1 \\
p_{1}(\xi)=A\left(\xi-\xi_{1}\right)^{m_{1}-1}\left(\xi-\xi_{2}\right)^{m_{2}-1} \cdots\left(\xi-\xi_{s}\right)^{m_{s}-1} h(\xi) \\
q_{1}(\xi)=\left(\xi-\eta_{1}\right)^{n_{1}+1}\left(\xi-\eta_{2}\right)^{n_{2}+1} \cdots\left(\xi-\eta_{t}\right)^{n_{t}+1}
\end{gathered}
$$

Since $g^{\prime}(\xi)+b\left(z^{\prime}\right)$ has a unique zero $\xi=\xi_{0}$, so we set

$$
g^{\prime}(\xi)+b\left(z^{\prime}\right)=\frac{B\left(\xi-\xi_{0}\right)^{l}}{\left(\xi-\eta_{1}\right)^{n_{1}+1} \cdots\left(\xi-\eta_{t}\right)^{n_{t}+1}}
$$

where $B$ is a nonzero constant. Then from (3.22), we have

$$
g^{\prime \prime}(\xi)=\frac{B\left(\xi-\xi_{0}\right)^{l-1} p_{2}(\xi)}{\left(\xi-\eta_{1}\right)^{n_{1}+2} \cdots\left(\xi-\eta_{t}\right)^{n_{t}+2}}
$$

where $p_{2}(\xi)=(l-q-t) \xi^{t}+b_{0} \xi^{t-1}+\cdots+b_{t-1}$ is a polynomial, $\operatorname{deg}\left(p_{2}\right) \leq t$.

From (3.21), it follow that

$$
g^{\prime \prime}(\xi)=\frac{A\left(\xi-\xi_{1}\right)^{m_{11}-2} \cdots\left(\xi-\xi_{s}\right)^{m_{s}-2} p_{3}(\xi)}{\left(\xi-\eta_{1}\right)^{n_{1}+2} \cdots\left(\xi-\eta_{t}\right)^{n_{t}+2}}
$$

where

$$
\begin{aligned}
p_{3}(\xi) & =(m-q)(m-q+1) \\
& \cdot \xi^{2 s+2 t-2}+c_{0} \xi^{2 s+2 t-3}+\cdots+c_{2 s+2 t-3}
\end{aligned}
$$

is also a polynomial, $\operatorname{deg}\left(p_{3}\right) \leq 2 s+2 t-2$.

We distinguish five cases to derivative a contradiction:

Subcase 1.1. $m=q$. Then from (3.21), we have $l=q+t$. So,

$$
\begin{gathered}
\operatorname{deg}\left(p_{2}\right)=t-i_{2}, 1 \leq i_{2} \leq t, \\
\operatorname{deg}(h)=s+t-1-h_{0}, 1 \leq h_{0} \leq s+t-1
\end{gathered}
$$

and

$$
\operatorname{deg}\left(p_{3}\right)=2 s+2 t-2-i_{3}, 1 \leq t_{3} \leq 2 s+2 t-2
$$

From (3.23) and (3.24), we have $i_{2}=i_{3}+1$. So also from (3.23) and (3.24), we also have $l-1 \leq \operatorname{deg}\left(p_{3}\right)$. Thus, we have $l \leq 2 s+2 t-1-i_{3}=2 s+2 t-i_{2}$.

Since $l=q+t$ and $q \geq 2 t$, then we have $t \leq 2 s-i_{2}$. On the other hand, from (3.23) and (3.24), we also have $m-2 s \leq \operatorname{deg}\left(p_{2}\right)$. Since $m \geq 4 s$, we have $2 s \leq t-i_{2}$. This is impossible.

Subcase 1.2. $m=q-1$. Then $l=q+t$,

$$
\operatorname{deg}\left(p_{2}\right)=t-i_{2}, \quad 1 \leq i_{2} \leq t, \operatorname{deg}(h)=s+t-1
$$

and

$$
\operatorname{deg}\left(p_{3}\right)=2 s+2 t-2-i_{3}, \quad 1 \leq t_{3} \leq 2 s+2 t-2
$$

Similarly to Subcase (1.1), from (3.23) and (3.24), we also have that $i_{2}=i_{3}+1$.

Also from (3.23) and (3.24), we have $l-1 \leq \operatorname{deg}\left(p_{3}\right)$, then, we have $t \leq 2 s+1-i_{2}$ On the other hand, similarly to the argument of Subcase (1.1), from (3.23) and (3.24), we also have $m-2 s \leq \operatorname{deg}\left(p_{2}\right)=t-i_{2}$, then $2 s \leq t-1-i_{2}$. This also is impossible.

Subcase 1.3. $m \leq q-2$. Then we still have $l=q+t \geq 3 t, \operatorname{deg}\left(p_{2}\right)=t-i_{2}, 1 \leq i_{2} \leq t, \operatorname{deg}(h)=s+t-1$, and $\operatorname{deg}\left(p_{3}\right)=2 s+2 t-2$. Therefor, $l \leq 2 s+2 t-2$, so $t \leq 2 s-2$. Similarly, we have $m-2 s \leq 2 s+t-i_{2}$, then $2 s \leq t-i_{2}$. This is a contradiction.

Subcase 1.4. $m=q+1$. Then $l \leq q+t$, $\operatorname{deg}(h)=s+t-1, \operatorname{deg}\left(p_{3}\right)=2 s+2 t-2$, and $\operatorname{deg}\left(p_{2}\right)=t-i_{2}, 0 \leq i_{2} \leq t$. From (3.23) and (3.24), we have $m \leq 2 s+t-i_{2}$. Thus, $2 s \leq t-i_{2}$ and $t \leq 2 s-1-i_{2}$. This is impossible.

Subcase 1.5. $m \geq q+2$. Then $l>q+t$, $\operatorname{deg}(h)=s+t-1, \operatorname{deg}\left(p_{3}\right)=2 s+2 t-2$, and $\operatorname{deg}\left(p_{2}\right)=t$. From (3.23) and (3.24), we have

$l-1 \leq \operatorname{deg}\left(p_{3}\right)=2 s+2 t-1$ and $m-2 s \leq \operatorname{deg}\left(p_{2}\right)=t$. So, we have that $t \leq 2 s-1$ and $2 s \leq t$. This is a contradiction.

Case 2. Suppose that there exists a complex number $\alpha \in C$ and a subsequence of sequence $\left\{z_{n} \rho_{n}^{-1}\right\}$, still noting it $z_{n} \rho_{n}^{-1}$, such that $z_{n} \rho_{n}^{-1} \rightarrow \alpha$. We have a con- 
verges

$$
\begin{aligned}
& H_{n}(\xi)=\rho_{n}^{-1} F_{n}\left(\rho_{n} \xi\right)=\rho_{n}^{-1} F_{n}\left(z_{n}+\rho_{n}\left(\xi-z_{n} / \rho_{n}\right)\right) \\
& \rightarrow g(\xi-\alpha)=\hat{g}(\xi)
\end{aligned}
$$

spherically uniform on compact subsets of $C$. Clearly, all zeros of $\hat{g}(\xi)$ are of multiplicity at least 4, all poles of $\hat{g}(\xi)$ are of multiplicity at least 2 . For each $\xi_{0} \neq 0$, it is easy to see that there exists a neighborhood $N\left(\xi_{0}, \delta\right)$ of $\xi_{0}$, such that $\xi^{r} H_{n}(\xi) \Rightarrow \xi^{r} \hat{g}(\xi)$, the convergence being spherically uniform on $N\left(\xi_{0}, \delta\right)$. For $\xi_{0}=0$, since $\xi_{0}$ is the pole of $g(\xi)$, then there exists $\delta>0$, such that $1 / \hat{g}(\xi)$ is analytic on $D_{2 \delta}=\{\xi:|\xi|<2 \delta\}$, $1 / H_{n}(\xi)$ are analytic on $D_{2 \delta}=\{\xi:|\xi|<2 \delta\}$ for sufficiently large $n$. Since

$$
1 / H_{n}(\xi)=\rho_{n} \xi^{r} f_{n}\left(\rho_{n} \xi\right)
$$

then $\xi_{0}=0$ is a zero of $1 / \hat{g}(\xi)$ has order at least $r$, we can deduce that $1 /\left(\xi^{r} H_{n}(\xi)\right)$ converges uniformly to $1 /\left(\xi^{r} \hat{g}(\xi)\right)$ on

$$
D_{\delta / 2}=\{\xi:|\xi|<\delta / 2\}
$$

Hence, we have

$$
G_{n}(\xi)=\frac{1}{\rho_{n}^{r+1} f_{n}\left(\rho_{n} \xi\right)}=\xi^{r} H_{n}(\xi) \rightarrow \xi^{r} \hat{g}(\xi)
$$

spherically uniform on compact subsets of $C$. It follows that $G(0) \neq 0$ from $f(\xi) \neq \infty$ whenever $a(\xi)=0$ for $\xi \in D$, hence all of zeros of $G(\xi)$ have order at least 4, all of poles of $G(\xi)$ have order at least 2. Noting that

$$
\begin{gathered}
\quad\left[G_{n}^{\prime}(\xi)+b\left(\rho_{n} \xi\right) \xi^{r}\right] G_{n}^{-2}(\xi)+\rho_{n}^{r+2} d\left(\rho_{n} \xi\right) \\
=\rho_{n}^{r+2}\left\{-f_{n}\left(\rho_{n} \xi\right)+a\left(\rho_{n} \xi\right) f_{n}^{2}\left(\rho_{n} \xi\right)+d\left(\rho_{n} \xi\right)\right\} \\
\rightarrow\left[G^{\prime}(\xi)+\xi^{r}\right] G^{-2}(\xi) \\
\text { If }\left[G^{\prime}(\xi)+\xi^{r}\right] G^{-2}(\xi) \equiv 0, \text { then } G^{\prime}(\xi)+\xi^{r} \equiv 0, \text { so } \\
\qquad G^{\prime}(\xi)=-\xi^{r}, G(\xi)=-\frac{\xi^{r+1}}{r+1}+C_{0}
\end{gathered}
$$

for any $\xi \in C$. Since $G(0) \neq 0$, then $C_{0} \neq 0$. Also since $G(\xi)$ has the zeros of multiplicity at least 4 , then $G(\xi) \neq 0$, this is a contradiction. Therefore,

$$
\left[G^{\prime}(\xi)+\xi^{r}\right] G^{-2}(\xi)
$$

is not identically equal to zero.

$$
\text { If }\left[G^{\prime}(\xi)+\xi^{r}\right] / G^{-2}(\xi) \text { for any } \xi \in C \text {, then } G(\xi)
$$
has no multiple poles and $G^{\prime}(\xi)+\xi^{r} \neq 0$. Note that
$G(\xi)$ has only multiple poles, so $G(\xi)$ is entire on $C$. Also by Lemma 2.5 , we have that $G(\xi)$ is not transcendental in $\mathrm{C}$, and then $G(\xi)$ is a polynomial. Thus, $G^{\prime}(\xi)=-\xi^{r}+C_{0}$, where $C_{0} \neq 0$. We have $G^{\prime \prime}(\xi)=-r \xi^{r-1}$, then from $G(0) \neq 0$ and a multiplicities of every zeros of $G(\xi)$ it follows that $G(\xi) \neq 0$ for any $\xi \in C$, this is impossible. Hence, $\left[G^{\prime}(\xi)+\xi^{r}\right] / G^{-2}(\xi)$ has some zeros. In fact, by the same argument as the Case 1 , we may deduce that $\left[G^{\prime}(\xi)+\xi^{r}\right] / G^{2}(\xi)$ has a unique zero $\xi=\xi_{0}$. Thus, we have that either $\xi=\xi_{0}$ is multiple poles of $G(\xi)$ or $\xi=\xi_{0}$ is a unique zero of $G(\xi)+\xi^{r}$.

Similarly, if $\xi=\xi_{0}$ is multiple poles of $G(\xi)$, from that $\left[G^{\prime}(\xi)+\xi^{r}\right] / G^{2}(\xi)$ has a unique zero $\xi=\xi_{0}$ it follows that $G^{\prime}(\xi) \neq-\xi^{r}$ for any $\xi \in C$. By Lemma 2.5, we have that $G(\xi)$ is not transcendental. Again by Lemma 2.6, we have that $G(\xi)$ is a constant, which is a contradiction. Hence, $G(\xi)$ has no multiple pole and $G^{\prime}(\xi)+\xi^{r}$ has a unique zero $\xi=\xi_{0}$. Thus, $G(\xi)$ is entire on $C$ and $G^{\prime}(\xi)+\xi^{r}$ has a unique zero $\xi=\xi_{0}$. By Lemma 2.5, we have that $G(\xi)$ must be a polynomial. Setting

$$
g(\xi)=A\left(\xi-\xi_{1}\right)^{m_{1}}\left(\xi-\xi_{2}\right)^{m_{2}} \cdots\left(\xi-\xi_{s}\right)^{m_{s}},
$$

where, $m_{1}, m_{2}, \cdots, m_{s}$ are $s$ positive integers, $m_{j} \geq 4$

$$
\begin{aligned}
& j=1,2, \cdots, s, m=\sum_{j=1}^{s} m_{j} \\
& G^{\prime}(\xi)+\xi^{r}=B\left(\xi-\xi_{0}\right)^{l},
\end{aligned}
$$

where $l$ is a positive integer, $l \geq 3$, we have

$$
\begin{gathered}
G^{\prime \prime}(\xi)+r \xi^{r-1}=B l\left(\xi-\xi_{0}\right)^{l-1}, \\
G^{(3)}(\xi)+r(r-1) \xi^{r-1}=B l(l-1)\left(\xi-\xi_{0}\right)^{l-2} .
\end{gathered}
$$

For $G(0) \neq 0$, we have $\xi_{0} \neq 0$ and $\xi_{j} \neq 0$. From (3.29) it follows that $\xi_{j} \neq \xi_{0}, j=1,2, \cdots, s$.

From (3.29), (3.30)and (3.31), for $j=1,2, \cdots, s$, we have

$$
\begin{gathered}
\xi_{j}^{r}=B\left(\xi_{j}-\xi_{0}\right)^{l} \\
r \xi_{j}^{r-1}=B l\left(\xi_{j}-\xi_{0}\right)^{l-1} \\
r(r-1) \xi_{j}^{r-2}=B l(l-1)\left(\xi_{j}-\xi_{0}\right)^{l-2}
\end{gathered}
$$

From (3.32) and (3.33), we have

$$
(r-l) \xi_{j}=r \xi_{0}, j=1,2, \cdots, s
$$

If $l=r$, then $\xi_{0}=0$, this is impossible. Therefore, we have $l \neq r$, and so 


$$
\xi_{1}=\xi_{2}=\cdots=\xi_{s}=\frac{r}{r-l} \xi_{0}
$$

From (3.33) and (3.34), we also have,

$$
\xi_{1}=\xi_{2}=\cdots=\xi_{s}=\frac{r-1}{r-l} \xi_{0}
$$

then $r \xi_{0}=(r-1) \xi_{0}$. Thus, we have $\xi_{0}=0$, a contradiction.

Finally, we prove that $F$ is normal at the origin. For any function sequence $\left\{f_{n}(z)\right\}$ in $F$, since $F_{1}$ is normal at $z=0$, then there exist a positive number $\delta<1 / 2$ and subsequence $\left\{F_{n_{k}}\right\}$ of $\left\{F_{n}\right\}$ such that $F_{n_{k}}$ converges uniformly to a meromorphic function $h(z)$ or $\infty$ on $N(0,2 \delta)$. Noting $F_{n}(0)=\infty$, we deduce that there exists a positive number $M>0$ such that $\left|F_{n_{k}}(z)\right| \geq M$ for any $z \in N(0, \delta)$. Again noting that $f_{n_{k}}(0) \neq \infty$ we have that $f_{n_{k}}(z) \neq \infty$ for all $z \in N(0, \delta)$, that is, $f_{n_{k}}(z)$ is analytic in $N(0, \delta)$. Therefore, for all $n_{k}$, we have

$$
\left|f_{n_{k}}(z)\right|=\left|\frac{1}{z^{r} F_{n_{k}}(z)}\right| \leq \frac{1}{M} \frac{2^{r}}{\delta^{r}},|z|<\frac{\delta}{2}
$$

By Montel's Theorem, $\left\{f_{n_{k}}(z)\right\}$ is normal at $z=0$, and thus $F$ is normal at $z=0$. The complete proof of Theorem 1.7 is given.

\section{Acknowledgements}

The authors are grateful to the referee for a number of helpful suggestions to improve the paper.

\section{References}

[1] W. Bergweiler and A. Eremenko, "On the Singularities of the Inverse to a Meromorphic Function of Finite Order," Revista Matemática Iberoamericana, Vol. 11, No. 2, 1995, pp. 355-373.
[2] H. H. Chen and M. L. Fang, "On the Value Distribution of $f^{n} f^{\prime}$," Science in China Series A, Vol. 38, No. 7, 1995, pp. 789-798.

[3] M. L. Fang and W. J. Yuan, "On the Normality for Families of Meromorphic Functions," Indian Journal of Mathematics, Vol. 43, 2001, pp. 341-350.

[4] W. K. Hayman, "Picard Values of Meromorphic Functions and the Its Derivatives," Annals of Mathematics, Vol. 70, 1959, pp. 9-42. doi:10.2307/1969890

[5] W. K. Hayman, "Meromorphic Functions," Clarendon, Oxford, 1964.

[6] X. J. Huang and Y. X. Gu, "Normal Families of Meromorphic Functions with Multiple Zeros and Poles," Journal of Mathematical Analysis and Applications, Vol. 295, No. 2, 2004, pp. 611-619. doi:10.1016/j.jmaa.2004.03.041

[7] X. J. Huang and Y. X. Gu, "Normal Families of Meromorphic Functions," Results in Mathematics, Vol. 49, 2006, pp. 279-288. doi:10.1007/s00025-006-0224-2

[8] S. Y. Li, "On Normal Criterion of Meromorphic Functions," Journal of Fujian Normal University, Vol. 25 1984, pp. 156-158.

[9] X. J. Li, "Proof of Hayman's Conjecture on Normal Families," Science in China Series A, Vol. 28, 1985, pp. 596-603.

[10] X. C. Pang, "On Normal Criterion of Meromorphic Functions," Science in China Series A, Vol. 33, No. 5, 1990, pp. 521-527.

[11] X. C. Pang, D. G. Yang and L. Zalcman, "Normal Families of Meromorphic Functions Omitting a Function ii," Computational Methods and Function Theory, Vol. 2, No. 1, 2002, pp. 257-265.

[12] Y. F. Wang and M. L. Fang, "Picard Values and Normal Families of Meromorphic Functions with Multiple Zeros," Acta Mathematica Sinica, Chinese Series, Vol. 41, No. 4, 1998, pp. 743-748.

[13] L. Zalcman, "Normal Families: New Perspectives," Bulletin (New Series) of the American Mathematical Society, Vol. 35, No. 3, 1998, pp. 215-230.

[14] Q. C. Zhang, "Normal Families of Meromorphic Functions Concerning Sharing Values," Journal of Mathematical Analysis and Applications, Vol. 338, No. 1, 2008, pp. 545-551. doi:10.1016/j.jmaa.2007.05.032 